\title{
Resistive Switching-based Electro-Optical Modulation
}

\author{
Enes Battal, Ayse Ozcan, and Ali Kemal Okyay*
}

For more than three decades, the mechanisms that optical modulation schemes relied on remained mainly the same. Here, we introduce a new modulation scheme based on resistive switching memories which is a non-volatile memory technology that became a very active research field. Exploiting the atomic scale modifications provided by the resistive switching effect, we show that optical modulation can be achieved starting from $4 \mu \mathrm{m}$ up to $18 \mu \mathrm{m}$ wavelengths which covers the spectral range of crucial applications such as bio-sensing, infrared spectroscopy and imaging and medical lasers. Through theoretical calculations and simulations following the optical measurements, we relate the modulation of optical constants to the variation in the effective doping concentration by the formation and rupture of filaments within the active layer. We confirm the variation of doping concentration reflected as a change in the local stoichiometry by energy dispersive X-ray spectroscopy and energy filtered transmission electron microscope measurements.

The workhorse of today's light engineering technologies for active photonic systems is optical modulators. Although optical modulation is heavily utilized for the realization of integrated optical interconnects to overcome the interconnect bottleneck in current complementary metal oxide semiconductor (CMOS) technology, recent innovations in photonics expand its applications, especially, in imaging, ${ }^{[1]}$ active matrix displays, ${ }^{[2]}$ biosensing, ${ }^{[3]}$ telecommunications, ${ }^{[4]}$ radio frequency and terahertz applications. ${ }^{[5]}$ Therefore, a momentum towards fast, high bandwidth, energy efficient, compact, scalable and integrable modulation technologies and devices has established. A wide variety of optical modulation schemes utilizing resonant ${ }^{[6]}$ or non-resonant $t^{[]}$effects providing improved control over electromagnetic properties of matter have been realized in recent years. However, the underlying mechanisms that provide optical modulation did not evolve much. Conventionally, optical

\section{E. Battal, Dr. A. K. Okyay}

Department of Electrical and Electronics Engineering UNAM - National Nanotechnology Research Center

Bilkent University

Ankara 06800, Turkey

E-mail: aokyay@ee.bilkent.edu.tr

E. Battal, A. Ozcan, Dr. A. K. Okyay

UNAM - National Nanotechnology Research Center Institute of Materials Science and Nanotechnology

Bilkent University

Ankara 06800, Turkey

A. Ozcan, Dr. A. K. Okyay

Department of Electrical and Electronics Engineering

UNAM - National Nanotechnology Research Center

Institute of Materials Science and Nanotechnology

Bilkent University

Ankara 06800, Turkey

DOI: 10.1002/adom.201400209 modulation is achieved utilizing plasma dispersion ${ }^{[8]}$ via electrical modulation of free carrier concentration in semiconductors. Thermal modulation is considered as an alternative due to availability of suitable materials for monolithic integration with large thermo-optic coefficients; ${ }^{[9]}$ yet, thermal stability requirements of the modulators bring additional operational costs. ${ }^{[4]}$ Hybrid schemes using liquid crystals ${ }^{[10]}$ for large index modulation, magneto-optical materials ${ }^{[11]}$ for fast switching, optically nonlinear materials ${ }^{[12]}$ for all optical modulation have also been demonstrated; however, with an additional penalty of elevated cost in fabrication process due to increased complexity of hybrid integration. Alternatively, solid-state phase-change materials ${ }^{[13]}$ switching between metallic and dielectric phases through atomic scale modifications have gained significant attention due to their high modulation contrast in refractive index, fast switching speeds, ${ }^{[14]}$ low switching power ${ }^{[15]}$ and non-volatility. ${ }^{[14]}$

Resistive switching memories, which are typically twoterminal non-volatile electrical devices operating by switching between two or more resistance states, are shown to exhibit similar atomic scale modifications, ${ }^{[16]}$ yet, unexplored for optical modulation. Recently, resistive switching memory devices are intensively studied due to natural simplicity in structure, fast switching speed, long retention time, low power consumption, suitability for 3D integration, excellent scalability below $20 \mathrm{~nm}$ and CMOS compatibility. ${ }^{[17]}$ Material-wise, resistive switching behavior is observed in almost all metal-oxides, as well as other dielectrics such as metal-nitrides, ${ }^{[18]}$ sulfides, ${ }^{[19]}$ fluorides, ${ }^{[20]}$ carbides $^{[21]}$ and phosphides. ${ }^{[22]}$ The atomic scale switching mechanism behind resistive switching is theorized to vary in different material systems. ${ }^{[23]}$ Several mechanisms have been suggested such that local capture and dispersion of filaments, ${ }^{[24]}$ ion transport recombination effect, ${ }^{[25]}$ space-charge-limited-current effect ${ }^{[26]}$ and modifications at the interface of the electrodes and the active region. ${ }^{[27]}$ Among these models, the formation of conductive filaments is very common and recently it has also been verified via in situ transmission electron microscopy (TEM) studies. ${ }^{[28]}$ Mechanisms based on atomic scale modifications that induce variations in extinction coefficient or refractive index of active device material, make resistive switching a good candidate as a promising optical modulation technique.

In this study, we introduce a novel electro-optic modulation method based on resistive switching effect by exploiting modifications at the atomic scale using electrical stimuli. Within the entire 5-18 $\mu \mathrm{m}$ wavelength spectrum, we obtain nonvolatile, hysteretic modulation of reflection spectrum up to $4 \%$. We obtain repeatable resistive switching behavior in electrical measurements and verify the formation of conducting filaments through transmission electron microscopy (TEM) images. We model the optical modulation by the modulation of effective doping concentration in the active layer due to the variation of local stoichiometry, verified by energy dispersive X-ray 
a)



b)

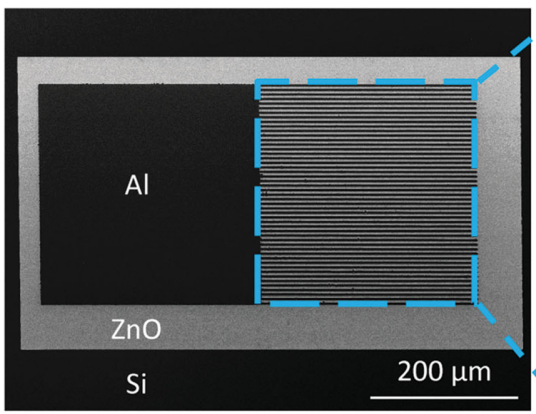

c)
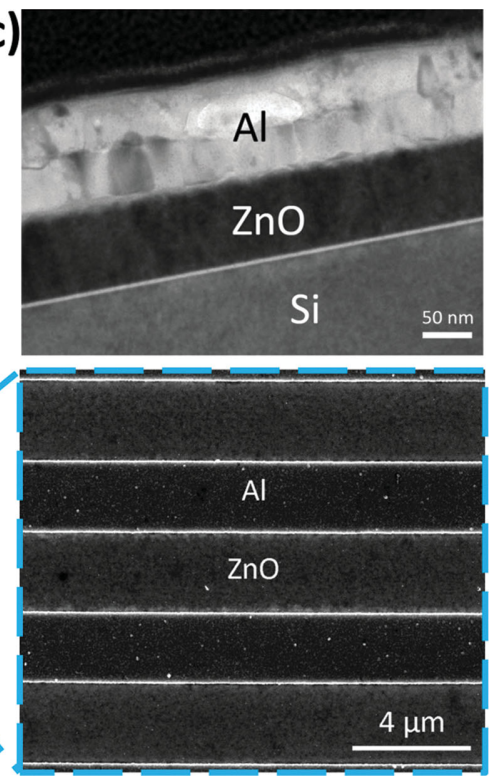

Figure 1. Depiction of the fabricated resistive switching memory along with SEM and TEM images. a) The resistive switching device is formed by sandwiching a semi-insulating $\mathrm{ZnO}$ film in between a metal (Al) and a highly conductive layer, p-type Silicon with resistivity of $3.1 \mathrm{~m} \Omega$ $\mathrm{cm}$. b) SEM image of the fabricated device shows the isolated $\mathrm{ZnO}$ layer and patterned top electrode. Top electrode is composed of a contact pad for electrical measurements and digitated fingers for optical measurements as shown in the magnified SEM image. c) Cross sectional TEM image of the virgin device depicts 80-nm-thick $\mathrm{ZnO}$ and 120 -nm-thick Al layers on Si. is applied from the top Al electrode while keeping bottom $\mathrm{Si}$ layer at zero volt. Initially, the virgin devices allow very small current flow, which is less than $100 \mu \mathrm{A} / \mathrm{cm}^{2}$. In order to observe resistive switching behavior, a controlled breakdown process, called electroforming, achieved by applying high electrical stress is essential. During this process, devices undergo atomic scale modifications as a result of high electrical bias and a current limitation is required to prevent permanent breakdown due to excess current flow. We perform electroforming by applying voltage bias of $-6 \mathrm{~V}$ for $1 \mathrm{~s}$ with a current compliance of $100 \mathrm{~mA}$. Conducting paths, namely filaments, are formed inside the active layer via electric field induced redox reactions ${ }^{[29]}$ and Joule heating ${ }^{[30]}$ as well as zinc and oxygen ion migration ${ }^{[31]}$; resulting in an increase in the overall conductivity of the films. The resistive switching behavior of the devices is achieved by varying voltage bias between -6 and $+6 \mathrm{~V}$ in dual sweep mode with the same current compliance. The I-V curve of the $\mathrm{Al} /$ $\mathrm{ZnO} / \mathrm{Si}$ device in Figure 2a exhibits a pinched hysteresis loop associated with resistive switching behavior. Starting with high resistance state (HRS), the device remains in this state during the voltage sweep from -6 to $6 \mathrm{~V}$ spectroscopy (EDX) and energy filtered transmission electron microscope (EFTEM) measurements. We also show modulation of overall electrical impedance consistent with our switching model. Up to 2 orders of magnitude modulation in effective doping concentration inside the active layer is predicted with our theoretical model.

Our resistive switching device, depicted in Figure 1a, is formed by consecutive depositions of $\mathrm{Al} / \mathrm{ZnO} / \mathrm{Si}$ stacked layers. Al layer forms the top electrode and the substrate is chosen as highly conductive p-type Silicon, to be used as a bottom electrode. Semiinsulating $\mathrm{ZnO}$ layer is patterned for device isolation and $\mathrm{Al}$ layer is patterned to form electrical contact pads and fingers for optoelectronic measurements. $\mathrm{Al}$ and $\mathrm{ZnO}$ layers have thicknesses of $120 \mathrm{~nm}$ and $80 \mathrm{~nm}$, respectively. Scanning electron microscope (SEM) images of the fabricated virgin device with a total area of $300 \times 600 \mu^{2}$ are shown in Figure 1b. Half of the device area consists of a square contact pad and the other half has fingers with width of $2.43 \mu \mathrm{m}$ and distance of $2.8 \mu \mathrm{m}$, whose corresponding transmission electron microscope (TEM) cross sectional image is depicted in Figure 1c.

Electrical characterization of $\mathrm{Al} / \mathrm{ZnO} /$ $\mathrm{Si}$ devices is performed through currentvoltage (I-V) measurements. Voltage bias

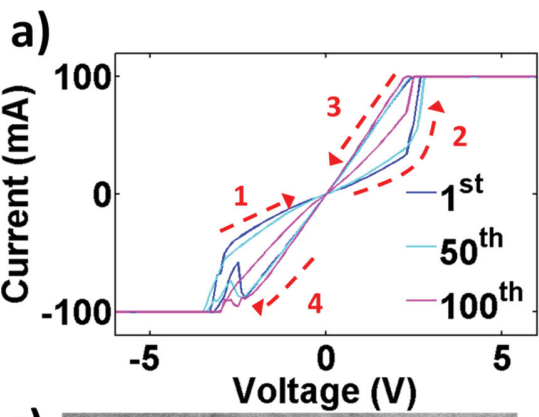

b)

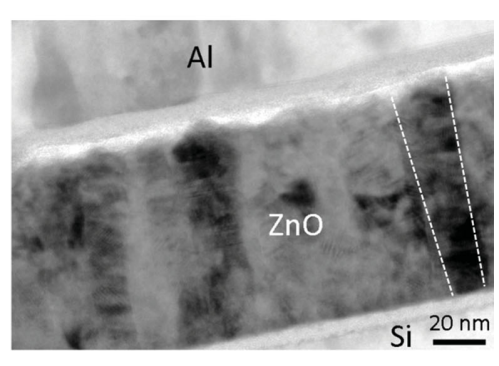

c)

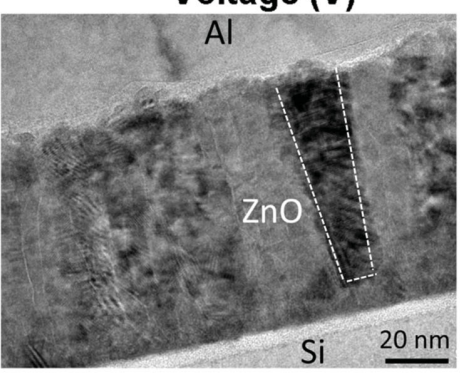

d)

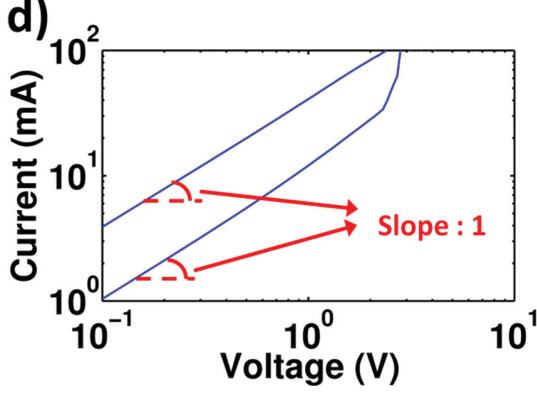

Figure 2. Electrical Characterization of the resistive switch memory device and TEM images at memory states. a) I-V characteristics of the resistive switching device showing repeatable hysteresis for over more than 100 cycles. $V_{\text {set }}$ and $V_{\text {reset }}$ values are observed to vary less than $0.3 \mathrm{~V}$. b) HRTEM cross sectional image shows a number of filaments reaching between the top and bottom electrodes after switching to LRS. c) HRTEM cross-sectional image at HRS shows partially dissolved filaments. d) For both LRS and HRS, log-log I-V curve gives a slope of 1 which indicates Ohmic conduction. 
the device switches to low resistance state (LRS) via set process. In Figure 2b, we show high-resolution transmission electron microscope (HRTEM) images of the device at LRS which depicts conducting filaments reaching from the top electrode to the bottom Si substrate. Considerable amount of current flow is due to the presence of these highly conductive paths. Triangular shapes of the filaments with their base on top Al layer are attributed to migration of oxygen ions towards the anode during electroforming process. ${ }^{[24]}$ As the voltage is swept from +6 to $-6 \mathrm{~V}$ for a device initially at LRS, an abrupt decrease in the current amplitude occurs at around $-2.4 \mathrm{~V}\left(\mathrm{~V}_{\text {reset }}\right)$ and the device switches back to HRS. Opposite polarity between $V_{\text {set }}$ and $V_{\text {reset }}$ indicate bipolar resistive switching characteristics. The energy consumption of the proposed scheme is vital, and depends on the filament density (see Supplementary Section S3) as well as filament formation and dissolution mechanism. The devices show switching behavior at $100 \mathrm{~mA}$ electrical current, and it may be possible to reduce this current level by using an alternative active material or reducing its thickness, however, such an investigation is not within the scope of this work. HRTEM cross-sectional image of the device at HRS in Figure 2c depicts partially dissolved filaments contributing to decrease in the conduction current while a considerable amount of fully conducting filaments still exists. The remaining conducting paths prevent the whole $\mathrm{ZnO}$ layer to behave like a good insulator; therefore, a low contrast ratio of 3 is achieved between two resistance states $\left(\mathrm{R}_{\mathrm{HRS}} / \mathrm{R}_{\mathrm{LRS}}\right)$. The device exhibits more than 100 repeated switching cycles between HRS and LRS. During these cycles, the device exhibits excellent stability since the set and reset voltages vary less than $0.3 \mathrm{~V}$ and the hysteresis curve does not change significantly as shown in Figure 2a. Retention times more than 5 days have been observed for the device at both states (See Supplementary Figure S1). For both states, the slope of log-log I-V curve shown in Figure 1d is ca. 1, which indicates a linear relationship between voltage and current; therefore, Ohmic conduction is dominant in the device. Ohmic behavior is attributed to the presence of through-reaching filaments in both LRS and HRS states ${ }^{[32]}$ which is confirmed by HRTEM images in Figure 2b,c. The atomic percentage of $\mathrm{Zn}$ is higher than $\mathrm{O}$ within a filamentary region of HRS and LRS devices verified by EDX results given in Table 1.

In order to investigate the effect of resistive switching on the optical characteristics of the device, we performed reflection measurements from the digitated device area shown in Figure 1b using a fourier transform infrared spectrometer (FTIR) while the device is electrically biased using the top contact pad and the bottom electrode. The typical size of the FTIR probe beam is ca $250 \times 250 \mu^{2}$, smaller than the device active area. A hysteretic behavior is observed in the reflection spectrum

Table 1. The atomic percentage of $\mathrm{Zn}$ and $\mathrm{O}$ within a filamentary and bulk region for HRS and LRS. The percentage values are calculated as the average atomic percentage of each atom on every measurement point along the line.

\begin{tabular}{lccccc}
\hline \multirow{2}{*}{ Atomic Percentage } & \multicolumn{2}{c}{ Filamentary Region } & & \multicolumn{2}{c}{ Bulk (No filamentary) Region } \\
\cline { 2 - 3 } \cline { 6 - 6 } & Zn [\%] & O [\%] & & Zn [\%] & O [\%] \\
\hline HR State & 52.16 & 47.82 & & 50.20 & 49.80 \\
LR State & 56.09 & 43.91 & & 46.35 & 53.65 \\
\hline
\end{tabular}

with applied voltage bias. A representative result of this hysteretic behavior is shown in Figure 3a which plots reflection at $8 \mu \mathrm{m}$ wavelength as a function of bias voltage (cycled between -6 and $+6 \mathrm{~V})$. Looping from 0 to $6 \mathrm{~V}$ at LRS, the change in the reflection is below $1 \%$ until a bias voltage of $4 \mathrm{~V}$. Right after $4 \mathrm{~V}$ of bias, a sudden drop in the reflection by $4 \%$ is observed. Looping back from 6 to $0 \mathrm{~V}$ does not change the reflection significantly at HRS. After going below $0 \mathrm{~V}$, reflection spectrum goes into a steady increase towards its original value at LRS. To observe the electrical switch a local small change in the stoichiometry is sufficient, however to observe optical switching whole bulk stoichiometry should be changed, so the optical switching observed higher voltage levels. The effect is verified to be nonvolatile such that the optical reflection state (LRS or HRS) stays at the last programmed state when the voltage bias is removed. Therefore, the resulting modulation in the reflection spectra is attributed to the change in the optical constants of $\mathrm{ZnO}$ layer due to atomic scale modifications caused by resistive switching. An ultra-broadband non-volatile modulation in the reflection within $5-18 \mu \mathrm{m}$ spectrum by $4 \%$ is observed at $0 \mathrm{~V}$ between HRS and LRS in Figure 3b.

Resistive switching phenomena is reported to modify local stochiometry within an oxide based active layer ${ }^{[24]}$ which would change effective doping concentration of the active layer; ${ }^{[33,34]}$ therefore modulate its effective dielectric permittivity. ${ }^{[35]}$ Modulation of doping concentration is known to significantly influence permittivity of doped semiconductors especially at longer wavelengths beyond the near infrared spectrum ${ }^{[35]}$ due to plasma dispersion. In order to account for the effects of doping, we measured the optical constants of $\mathrm{n}$ doped $\mathrm{ZnO}$ films with doping concentrations ranging from $10^{16} \mathrm{~cm}^{-3}$ up to $10^{21} \mathrm{~cm}^{-3}$ and performed finite difference time domain simulations (FDTD) using these values to compare with the measurements. Dielectric values of $\mathrm{ZnO}$ films are fit using Supplementary Equation (1) which includes Drude oscillator formulation to account for plasma dispersion of free carriers. For the optical constants of the underlying silicon layer below $\mathrm{ZnO}$, literature data ${ }^{[36]}$ for boron doped silicon with resistivity of $3.1 \mathrm{~m} \Omega-\mathrm{cm}$ is used. Doping concentrations of $3.84 \times 10^{19}$ for HRS and $4.4 \times 10^{19} \mathrm{~cm}^{-3}$ for LRS (dielectric parameters are given in Supplementary Table 1), yielded best fitting results, and corresponding refractive index values are plotted in Figure 3c. For both LRS and HRS states, the simulated reflection spectra in Figure 3d shows close resemblance with the FTIR reflection measurements. The results show higher reflection for high doping level (LRS) compared to low doping level (HRS) with more than $4 \%$ reflection modulation within 5-18 $\mu \mathrm{m}$ spectrum. The contrast in the reflection between HRS and LRS, for the wavelengths lower than $5 \mu \mathrm{m}$, remains below $1 \%$ in agreement with the measured results. Comparing the simulated electric field intensity profiles at $4 \mu \mathrm{m}$ and $14 \mu \mathrm{m}$ in Figure $3 \mathrm{e}$ and $\mathrm{f}$, respectively, stronger localization within the $\mathrm{ZnO}$ layer is clear at higher wavelengths. Therefore, the larger modulation contrast of the reflection spectrum for wavelengths above $5 \mu \mathrm{m}$ can be attributed to the stronger interaction of the electric field with the $\mathrm{ZnO}$ layer at higher wavelengths. In addition, the contrast in the reflection spectra at wavelengths above $18 \mu \mathrm{m}$ decreases for the theoretical doping modulation model in agreement with measurements. Agreement between FDTD simulations 

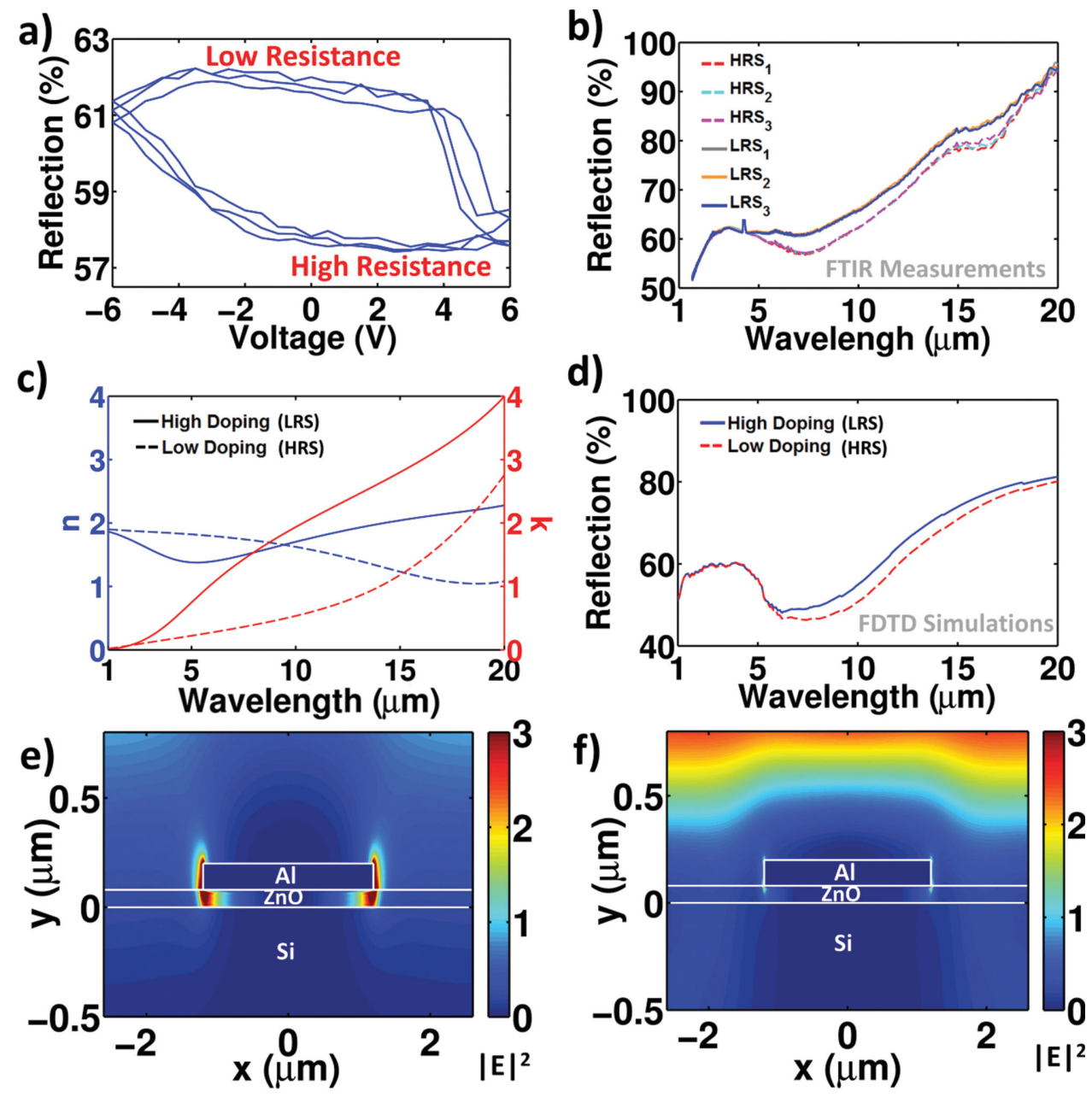

Figure 3. Optoelectronic characterization and FDTD modelling of the resistive switch device. Optical hysteresis is observed in a) reflection at the wavelength of $8 \mu \mathrm{m}$ as a function of bias voltage. (See Supplementary S2 for other wavelengths) b) A non-volatile modulation of reflection by $4 \%$ in the 5-18 $\mathrm{mm}$ range is observed in the FTIR reflection spectrum, measured at OV. The subscripts denote cycle number of the refection spectra. c) Simulated variation of optical constants for effective doping concentrations of $4.4 \times 10^{19} \mathrm{~cm}^{-3}$ and $3.84 \times 10^{19} \mathrm{~cm}^{-3} \mathrm{~d}$ ) Simulated reflection spectra, taking modulation of doping concentration during switching into account, agrees well with the FTIR measurements. e) Electric field intensity profile for the wavelength of $14 \mu \mathrm{m}$ shows stronger localization of the incident electromagnetic field within $\mathrm{ZnO}$ layer than f) the case for $4 \mu \mathrm{m}$. Therefore, higher interaction with the $\mathrm{ZnO}$ layer results in higher modulation in the reflection at higher wavelengths.

including doping modulation and FTIR measurements strongly supports the doping (free carrier) modulation model due to cyclic rupture and dissolution (HRS) and reformation (LRS) of filaments during resistive switching.

Capacitance value of a two terminal device is known to be quite sensitive to localized morphological changes within the active layer as opposed to its resistance value. Especially, variation in the dielectric parameters would reflect on the measured device capacitance. Therefore, we performed capacitance measurements of our two terminal resistive switching memory device to analyze the underlying transient non-volatile modifications. A cyclic voltage bias between -6 and $+6 \mathrm{~V}$ resulted in nonvolatile hysteretic capacitance values shown in Figure 4a. For an active layer formed by a semiconductor, capacitance value is expected to increase with doping concentration. ${ }^{[37]}$ Hence, the measured capacitance values are higher at the LRS (highly doped) compared to those at the HRS (lowly doped). I-V measurements show sudden switching into LRS, for instance, when
Zn-rich filament forms a conductive path. When measuring capacitance, on the other hand, a gradual transition is observed since measured capacitance is determined by the effective dielectric medium, where $\mathrm{Zn}$ and $\mathrm{O}$ atoms are gradually migrating during the swithing.

Energy filtered transmission electron microscopy (EFTEM) images demonstrating oxygen concentrations in Figure $4 \mathrm{~b}$ obtained from a filament region of the device shows lower oxygen concentration within a filament compared to bulk regions and supports the variation in local stoichiometry within ZnO films. To understand the transition between HRS and LRS resulting in electro-optic modulation in our $\mathrm{Al} / \mathrm{ZnO} / \mathrm{Si}$ resistive switching device, we performed energy dispersive X-ray spectroscopy (EDX) measurements of the filamentary regions at HRS and LRS. A representative EDX measurement location is indicated on TEM cross section in Figure 4c. A clear contrast in $\mathrm{Zn} / \mathrm{O}$ stoichiometry within the filamentary region is observed between LRS and HRS. Difference in the average Zn 


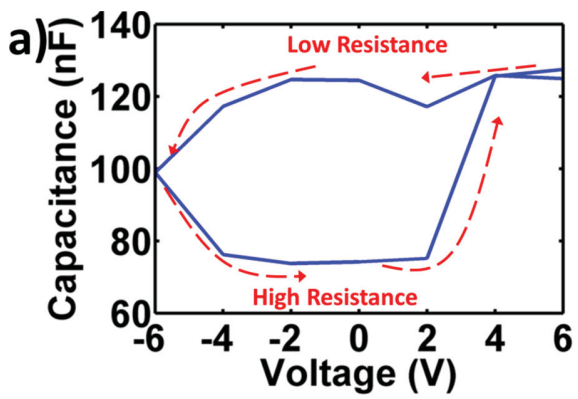

\section{b)}

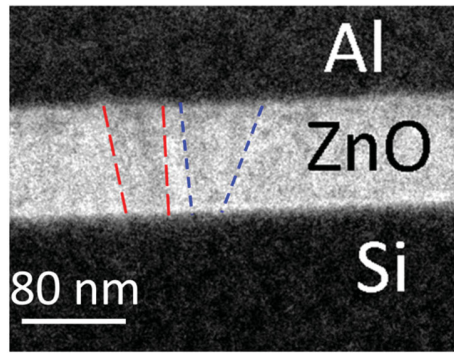

c)

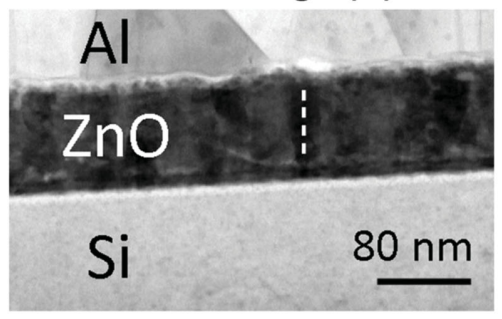

d)

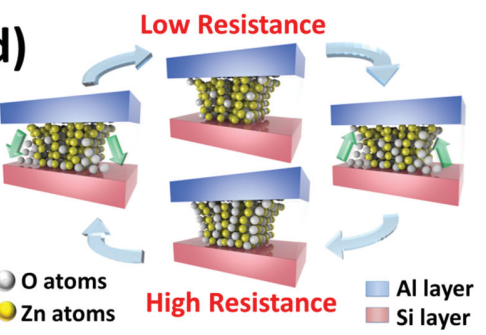

Figure 4. Ion migration based modulation mechanism. a) The hysteretic loop in the capacitance of $\mathrm{Al} / \mathrm{ZnO} / \mathrm{Si}$ structure as a function of bias voltage shows non-volatile modulation of the dielectric values within the device. b) EFTEM images reveal oxygen deficiency within a filament compared to bulk regions which indicates variation of local stoichiometry within the device. c) TEM image indicating the region of EDX measurement along filaments at HRS and LRS, which are performed to quantify to variation in oxygen concentration between those states. d) The resulting model depicting migration and return of the oxygen atoms around the filaments, which results in modulation of doping concentration; therefore, optical constants.

to $\mathrm{O}$ concentration on filamentary regions with respect to nonfilamentary regions in terms of signal counts in EDX spectroscopy is measured to be $5 \%$ for LRS whereas it is about $1.85 \%$ for HRS indicating higher stoichiometry for HRS. For thin film $\mathrm{ZnO}$, zinc interstitials ${ }^{[33]}$ or oxygen vacancies ${ }^{[34]}$ are reported to account for unintentional doping. These findings also support that electro-optical modulation observed in the FTIR measurements are caused by variation of effective doping caused by variation of oxygen concentration inside the filaments between HRS and LRS. The complete mechanism, depicted in Figure 4d, is consistent with our observations in FTIR, C-V, EFTEM and EDX measurements.

In conclusion, we demonstrated resistive switching as a new and viable optical modulation technique. By forming $\mathrm{Al} / \mathrm{ZnO} / \mathrm{Si}$ structure, we achieved a proof-of-principle nonvolatile and repeatable resistive switching characteristics. Through FTIR reflection measurements, we showed optical modulation of reflection by $4 \%$ within the spectral range covering nearly the entire mid and long-wave infrared wavelengths. The involvement of the filament formation and rupture in the resistive switching mechanisms is observed via TEM images. The resulting optical modulation is modeled via considering change of effective doping concentration within the active layer due to variation of local stoichiometry. The performed capacitance measurements indicating variation of dielectric parameters exhibit hysteretic behavior similar to that of the resistive switching characteristics. EFTEM and EDX results support the variation of local stoichiometry within the filamentary regions in agreement with our model. The results of this work bridge resistive switching mechanism to optical modulation and enable alternative modulation schemes such as reconfigurable non-volatile surfaces, imagers, emitters as well as electro-optic memories.

\section{Experimental Section}

Device Fabrication: Device fabrication starts on a highly doped $(3.1 \mathrm{~m} \Omega \mathrm{cm}$ ) silicon (100) substrate. After standard RCA wafer cleaning, a 80-nm-thick $\mathrm{ZnO}$ layer is deposited at room temperature by reactive magnetron (RF) sputtering using a $\mathrm{ZnO}$ target having $99.999 \%$ purity at $150 \mathrm{~W}$ RF power and 20 mTorr Ar pressure under vacuum condition. $\mathrm{ZnO}$ is patterned with optical lithography and etching in dilute $(2.5 \%) \mathrm{H}_{2} \mathrm{SO}_{4}$ solution. Then, the films are plasma annealed at $125^{\circ} \mathrm{C}$ with $30 \mathrm{sccm} \mathrm{O} \mathrm{O}_{2}$ and $5 \mathrm{sccm} \mathrm{N} \mathrm{N}_{2}$ gas flow. 120-nm-thick Al contact pad and finger layer is formed by thermal evaporation followed by lift-off process.

Electrical Characterization: Electrical measurements are performed using Agilent B1500A semiconductor parameter analyzer. A voltage loop between -6 and $+6 \mathrm{~V}$ is applied with $0.1 \mathrm{~V}$ steps and $100 \mathrm{~ms}$ hold and wait times. The resistance contribution from the measurement equipment is measured to be less than $0.1 \Omega$; therefore, it is ignored in the measurements. Capacitance measurements are carried out using Keithley 4200 C-V measurement unit coupled with Cascade Microtech PM-5 probe station at $200 \mathrm{KHz}$ frequency with $30 \mathrm{mV}$ rms alternating voltage. C-V measurements are performed in a non-volatile manner such that, at each step, initially a dc voltage bias is applied and the capacitance is measured after the DC biased is temporarily removed.

Optoelectronic Characterization: Optoelectronic characterization of the device is performed using Bruker Vertex 70 Fourier Transform Infrared Spectrometer with Hyperion 2000 Microscope attachment. We coupled the microscope with a Keithley 2400 source measure unit and electrical probes for simultaneous reflection measurements under electrical bias. The measurements are performed with $0.5 \mathrm{~V}$ bias steps and $55 \mathrm{~s}$ hold time to compensate for the long measurement duration of the spectrometer. Custom-made automated software is used to sweep the bias voltage and monitor the resistance state while performing reflection measurements at each bias step.

FDTD Simulations: FDTD simulations are performed using a commercial package, FDTD Solutions by Lumerical Inc. Due to presence of oblique injection up to $10^{\circ}$ within our measurement setup; we performed simulations for each angle of incidence up to $10^{\circ}$ with $2^{\circ}$ steps and averaged out the results to obtain the simulated reflection spectra. Bloch boundary conditions are used for x-axis to simulate the replicated fingers within a unit-cell correctly for non-oblique incidence. Perfectly matching boundary conditions are employed for $y$-axis. Mesh size of $10 \mathrm{~nm}$ is used throughout the structure.

TEM Images: Different fresh/virgin devices are configured to different resistance states and TEM samples are prepared by taking crosssectional slices from active device area with fingers. Sample preparation is performed using FEI Nova 600i Nanolab Focused Ion Beam Milling machine. TEM imaging studies are carried out using FEI Tecnai G2 F30 TEM device.

\section{Supporting Information}

Supporting Information is available from the Wiley Online Library or from the author. 


\section{Acknowledgements}

This work was supported by the Scientific and Technological Research Council of Turkey (TUBITAK), grant numbers 109E044, 112M004, $112 \mathrm{E} 052$ and $113 \mathrm{M} 815$. A. K. O. acknowledges a Marie Curie International Reintegration Grant (PIOS, Grant \# PIRG04-GA-2008-239444). E. B. acknowledges TUBITAK-BIDEB for national M.Sc. fellowships. We thank Mustafa Guler for the preparation of TEM samples and performing HRTEM, EFTEM and EDX measurements.

Received: May 5, 2014 Revised: July 28, 2014 Published online:

[1] A. Garcia Sega, B. H. King, J. Y. Lee, M. J. Sailor, G. M. Miskelly, ACS Nano 2013, 7, 7785.

[2] D. Fattal, Z. Peng, T. Tran, S. Vo, M. Fiorentino, J. Brug, R. G. Beausoleil, Nature 2013, 495, 348.

[3] H. Kang, C.-J. Heo, H. C. Jeon, S. Y. Lee, S.-M. Yang, ACS Appl. Mater. Interfaces 2013, 5, 4569.

[4] G. T. Reed, G. Mashanovich, F. Y. Gardes, D. J. Thomson, Nat. Photon. 2010, 4, 518.

[5] B. Sensale-Rodriguez, R. Yan, M. M. Kelly, T. Fang, K. Tahy, W. S. Hwang, D. Jena, L. Liu, H. G. Xing, Nat. Commun. 2012, 3, 780.

[6] I. M. Pryce, K. Aydin, Y. A. Kelaita, R. M. Briggs, H. A. Atwater, Nano Lett. 2010, 10, 4222

[7] R. M. Briggs, I. M. Pryce, H. A. Atwater, Opt. Express 2010, 18, 11192.

[8] R. A. Soref, B. R. Bennett, IEEE J. Quantum Electron. 1987, 23, 123.

[9] J. Komma, C. Schwarz, G. Hofmann, D. Heinert, R. Nawrodt, Appl. Phys. Lett. 2012, 101, 041905.

[10] J. Pfeifle, L. Alloatti, W. Freude, J. Leuthold, C. Koos, Opt. Express 2012, 20, 15359.

[11] V. V. Temnov, Nat. Photon. 2012, 6, 728.

[12] R. B. Li, L. Deng, E. W. Hagley, Phys. Rev. Lett. 2013, 110, 113902.

[13] Y. Lu, L. Liu, D. Mandler, P. S. Lee, J. Mater. Chem. C 2013, 1, 7380.

[14] Y. C. Yang, F. Pan, Q. Liu, M. Liu, F. Zeng, Nano Lett. 2009, 9, 1636.

[15] L. Heng-Yuan, C. Pang-Shiu, W. Ching-Chiun, M. Siddheswar, T. Pei-Jer, L. Cha-Hsin, L. Lurng-Shehng, T. Ming-Jinn, Jpn. J. Appl. Phys. 2007, 46, 2175.

[16] R. Waser, M. Aono, Nat. Mater. 2007, 6, 833.

[17] a) M.-J. Lee, C. B. Lee, D. Lee, S. R. Lee, M. Chang, J. H. Hur, Y.-B. Kim, C.-J. Kim, D. H. Seo, S. Seo, U. I. Chung, I.-K. Yoo,
K. Kim, Nat. Mater. 2011, 10, 625; b) H. Akinaga, H. Shima, Proc. IEEE 2010, 98, 2237; c) S. H. Jo, W. Lu, Nano Lett. 2008, 8, 392; d) S. Yu, H.-Y. Chen, B. Gao, J. Kang, H. S. P. Wong, ACS Nano 2013, 7, 2320.

[18] B. Choi, J. J. Yang, M. X. Zhang, K. Norris, D. A. Ohlberg, N. Kobayashi, G. Medeiros-Ribeiro, R. S. Williams, Appl. Phys. A 2012, 109, 1.

[19] A. Geresdi, M. Csontos, A. Gubicza, A. Halbritter, G. Mihaly, Nanoscale 2014, 6, 2613.

[20] B. F. Bory, H. L. Gomes, R. A. J. Janssen, D. M. de Leeuw, S. C. J. Meskers, J. Phys. Chem. C 2012, 116, 12443.

[21] L. Wootae, P. Jubong, S. Myungwoo, L. Joonmyoung, J. Seungjae, K. Seonghyun, P. Sangsu, S. Jungho, H. Hyunsang, IEEE Electron Device Lett. 2011, 32, 680 .

[22] S. Balasubramanian, V. Kumar, N. Balasubramanian, V. Premachandran, Appl. Phys. Lett. 1994, 64, 2256.

[23] J. J. Yang, D. B. Strukov, D. R. Stewart, Nat. Nano. 2013, 8, 13.

[24] D.-H. Kwon, K. M. Kim, J. H. Jang, J. M. Jeon, M. H. Lee, G. H. Kim, X.-S. Li, G.-S. Park, B. Lee, S. Han, M. Kim, C. S. Hwang, Nat. Nano. 2010, 5, 148.

[25] B. Gao, J. Kang, L. Liu, X. Liu, B. Yu, Appl. Phys. Lett. 2011, 98, 232108

[26] Y. Xia, W. He, L. Chen, X. Meng, Z. Liu, Appl. Phys. Lett. 2007, 90, 022907.

[27] H. Y. Hwang, Y. Iwasa, M. Kawasaki, B. Keimer, N. Nagaosa, Y. Tokura, Nat. Mater. 2012, 11, 103.

[28] a) J.-Y. Chen, C.-L. Hsin, C.-W. Huang, C.-H. Chiu, Y.-T. Huang, S.-J. Lin, W.-W. Wu, L.-J. Chen, Nano Lett. 2013, 13, 3671; b) Y. Yang, P. Gao, S. Gaba, T. Chang, X. Pan, W. Lu, Nat. Commun. 2012, 3, 732.

[29] R. Waser, R. Dittmann, G. Staikov, K. Szot, Adv. Mater. 2009, 21, 2632.

[30] U. Russo, D. Ielmini, C. Cagli, A. L. Lacaita, IEEE Trans. Electron Devices 2009, 56, 193.

[31] R. Yang, K. Terabe, G. Liu, T. Tsuruoka, T. Hasegawa, J. K. Gimzewski, M. Aono, ACS Nano 2012, 6, 9515.

[32] K. M. Kim, B. J. Choi, Y. C. Shin, S. Choi, C. S. Hwang, Appl. Phys. Lett. 2007, 91, 012907.

[33] K. Hoffmann, D. Hahn, Phys. Status Solidi 1974, 24, 637.

[34] A. Pöppl, G. Völkel, Phys. Status Solidi 1991, 125, 571.

[35] C. A. Wolden, T. M. Barnes, J. B. Baxter, E. S. Aydil, J. Appl. Phys. 2005, 97, 043522.

[36] S. Basu, B. J. Lee, Z. M. Zhang, J. Heat Transfer 2009, 132, 023301.

[37] C. van Opdorp, Solid-State Electron. 1968, 11, 397 